\title{
LETTER
}

\section{Greater care needed}

Sir, We are writing in response to the recent feature, 'Where are all the specialists?' $\mathrm{We}$ appreciate the editor was looking to draw attention to the shortage of specialists of all types and we wholeheartedly support the continued highlighting of this important issue.

As with all the specialisms, there is a lack of funding at the heart of the matter. There is a shortage of all dentists and orthodontics is not immune from this. The question is asked, 'How can in excess of 1,400 specialists result in waiting lists?' The answer is simple - because it is the commissioners who determine how much NHS orthodontic treatment is provided. The number of UOAs on offer is determined by NHS England/NHS Wales.

In contrast, Scotland and Northern Ireland do not have contracts with UOAs but instead still use 'item of service' payments to fund orthodontic care. Waiting lists for primary care NHS orthodontic treatment are not an issue in these countries.

The decision taken to change the contracting process reducing or removing completely the number of UOAs, in England and Wales, has forced many specialists who were previously quite happily providing NHS orthodontic treatment to now provide private treatment simply to maintain income.

The huge growth in private orthodontics alluded to in recent years has been largely driven by GDPs. Many provided orthodontic treatment under the NHS but now cannot compete for an orthodontic contract.

We are concerned that there appears to be a number of misunderstandings of how career pathways in orthodontics work. All training pathways are clear and transparent on GDC, Royal College of Surgeons and BOS websites. Recent surveys have shown that the majority of training grades desire to have part time consultancy - this reflects a work/life balance shift that we suspect will be reflected across all specialisms as full-time consultancies become rarer.

Three years post-graduate orthodontic training, qualifies for inclusion on the specialist list and, allows the orthodontist to work in specialist practice. $90-95 \%$ of NHS orthodontic treatment in England and Wales is provided in primary care. This is what commissioners are aiming for.

There may be a perception that a large amount of private work is being carried out by these individuals, but it is similar to general dental practice. Working in your own business may be deemed to be 'private' but ignores the fact that many will have a large NHS commitment. To qualify as a consultant two further years of extra training is undertaken but this doesn't confine you to the NHS. Many choose part time NHS consultancy and part time practice, as alluded to above.

We also would suggest that greater care with the use of language in the piece could have been taken as it seemed in some sections of the feature that orthodontics was being unfairly singled out for criticism. We feel that with the high levels of stress that many orthodontists are currently under, due to a myriad of reasons including: retendering, loss of contracts and massive reductions in UOA to name just three, language such as 'heading off to the private sector in search of more money', 'are specialists following the money' and 'is there a move towards work that has more of a financial gain' is wholly inappropriate.

P. McCallum, BOS, via email

\section{Reference}

1. Westgarth $\mathrm{D}$. Where are all the specialists? BDJ In Pract 2020; 33: 12-16.

\section{BDA Indemnity supports members with immediate cost reductions of up to $80 \%$}

The British Dental Association will continue to offer unparalleled support and flexibility for its members during the COVID-19 pandemic, enabling members to immediately reduce rates while maintaining all of the benefits, protections and security of the RSA-underwritten policy.

The adjusted rates reflect the dramatic changes in working hours and workload of dentists, and typically represent savings of $70-80 \%$ for associates and $65-70 \%$ for

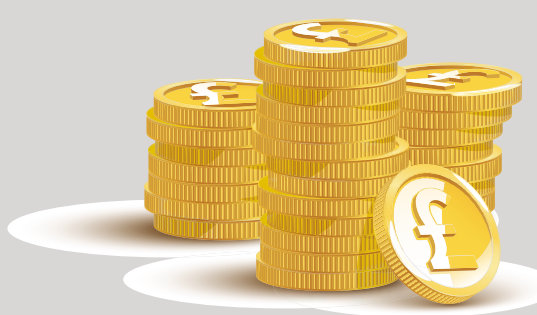

practice owners who were previously working the equivalent of three days or more per week.

Len D'Cruz, head of the BDA's indemnity team said: 'We're in uncharted waters. A dentist leaving themselves without any cover right now is sailing without a life jacket.

'It's fundamental to us that our members should be able to see all the benefits of their cover through and beyond this emergency, while paying lower rates to fairly reflect their changed circumstances.

'We have an occurrence-based policy. So, while we're all working out what form of practice we'll be doing in the coming months, whether it's triage from home or on the frontline at an emergency clinic, it's more important than ever to have contractual indemnity cover. A practice owner whose staff are taking calls and doing their best to advise patients will still want vicarious liability protection.

'In a crisis like this the value of the BDA's indemnity policy has never been more obvious. As part of the BDA family we can offer members real peace of mind, up to the minute advice and the certainty that we'll be fighting their corner.

The policy covers GDC investigations, challenges by a wide range of other regulators, HMRC investigations and adverse media coverage all of which might still be called upon in clinically fallow times.

Members with policies can request their cover to be amended by emailing us details of their situation. Please email us on bda. indemnity@bda.org. For details of the policy please visit bda.org/indemnity/policy 